\title{
Transcriptional repression by the basic helix-loop-helix protein Dec2: Multiple mechanisms through E-box elements
}

\author{
KATSUMI FUJIMOTO ${ }^{1}$, HIDENORI HAMAGUCHI ${ }^{1,2}$, TAKAFUMI HASHIBA ${ }^{1}$, TADAHIRO NAKAMURA ${ }^{1}$, \\ TAKESHI KAWAMOTO ${ }^{1}$, FUYUKI SATO ${ }^{1,3}$, MITSUHIDE NOSHIRO ${ }^{1}$, UJJAL K. BHAWAL ${ }^{1}$, \\ KETUT SUARDITA $^{1}$ and YUKIO KATO ${ }^{1}$
}

\begin{abstract}
Departments of ${ }^{1}$ Dental and Medical Biochemistry, ${ }^{2}$ Periodontal Medicine, Hiroshima University Graduate School of Biomedical Sciences, 1-2-3 Kasumi, Minami-ku, Hiroshima 734-8553; ${ }^{3}$ Department of Pathology,

Hirosaki University School of Medicine, 5-zaifu-cho, Hirosaki 036-8562, Japan
\end{abstract}

Received September 4, 2006; Accepted October 30, 2006

\begin{abstract}
Dec2, a member of the basic helix-loop-helix (bHLH) superfamily, has been shown to function as a transcriptional repressor and is implicated in cell proliferation and differentiation. In addition, Dec2 transcripts exhibit a striking circadian oscillation in the suprachiasmatic nucleus. To identify the molecular mechanisms by which Dec2 regulates gene expression, we carried out structure-function analyses. Gel retardation and luciferase assays showed that Dec2, as well as its related protein Dec1, preferentially binds to class B E-box elements (CACGTG) as a homodimer and represses the transcription of target genes in a histone deacetylase (HDAC)-dependent manner. Functional studies with the GAL4-DNA binding domain fusion proteins identified the domain responsible for the repression activity of Dec2 in its C-terminal region, which is also necessary to recruit HDAC1. In addition, the basic and HLH domains of Dec2 were required for DNA binding and homodimerization, respectively. In contrast, Dec proteins repressed a MyoD-activated promoter activity of muscle creatine kinase gene through class A E-box in an HDAC1-independent manner. Dec2 formed a heterodimer with MyoD through the basic and HLH domains. Consistent with this, both the basic and HLH domains were required for the ability of Dec2 to inhibit the transcriptional activity of MyoD. These findings indicate that Dec2 employs multiple mechanisms, including DNA-binding and proteinprotein interactions, to achieve E-box-dependent transcriptional repressions.
\end{abstract}

Correspondence to: Dr Yukio Kato, Department of Dental and Medical Biochemistry, Hiroshima University Graduate School of Biomedical Sciences, 1-2-3 Kasumi, Minami-ku, Hiroshima 7348553, Japan

E-mail: ykato@hiroshima-u.ac.jp

Key words: Dec2, Dec1, E-box, MyoD, histone deacetylase

\section{Introduction}

The basic helix-loop-helix (bHLH) transcription factors make up a large family of proteins that regulate a wide variety of biological processes, including proliferation and differentiation (1). These proteins, forming homodimers or heterodimers, regulate the expression of target genes by binding to the E-box consensus sequence (CANNTG). The basic region has been shown to be necessary for specific DNA binding, whereas the HLH domain mediates dimerization. These transcription factors are functionally classified into two groups, transcriptional activators and transcriptional repressors. Members of the bHLH-Orange (bHLH-O) proteins are known to be repressive transcription factors, and these proteins share a common sequence motif, the Orange domain, located just at the C-terminal to the bHLH domain. Based on their primary structures, the bHLH-O family is subdivided into five classes. The Hairy, Enhancer of Split, Hey (also named HRT, Hesr, CHF, HERP, and Gridlock), Dec (also named Stra13 and Sharp), and Helt subfamilies $(2,3)$. Among the bHLH-O proteins, Hes1 is most strongly characterized. A homodimer of Hes1 binds to the class B E-box (CANGTG), class C E-box (CANGCG), and N-box (CACNAG) and represses the transcription of target genes (2). Hes 1 also antagonizes the transcriptional activity of MyoD by direct interaction and inhibits MyoD-induced myogenesis (2).

Dec1 and Dec2 form a subfamily within bHLH-O proteins (4-7). During mouse embryogenesis, these genes are expressed in many tissues, including the brain, heart, liver, and limb bud, suggesting that they play important roles in the development of ectodermal, mesodermal, and endodermal lineages, and overexpression of Dec1 does indeed induce the differentiation of nerve cells and chondrocytes $(6,8)$. Dec1 and Dec2 also appear to function as negative regulators of adipogenesis and myogenesis $(9,10)$, respectively. Furthermore, Dec1-deficient mice exhibit ineffective elimination of activated $\mathrm{T}$ and $\mathrm{B}$ cells (11). Interestingly, transcripts of Dec1 and Dec2 exhibit a striking circadian oscillation in the suprachiasmatic nucleus, suggesting that they function as regulatory proteins for the clockwork system (12). Collectively, these findings demonstrated that Dec family proteins are involved in many biological 
processes. Dec proteins are known to interact with their promoter through the CACGTG E-box, and therefore repress their own transcription $(13,14)$, but the molecular mechanism by which these proteins, particularly Dec2, function has not been examined extensively.

In this study, we found that Dec2 interacts with HDAC1 and SIRT1, and that HDAC1 is involved in the Dec2-mediated direct repression but not in the Dec2-mediated inhibition of MyoD activity. In addition, we performed functional studies on the Dec2 domains.

\section{Materials and methods}

Plasmids. The mammalian expression vectors pcDNA$m D e c 1$ and pcDNA-mDec2 were described previously (14). The $m$ Dec 2 deletion mutants were generated by PCR followed by self-ligation. The following pairs of oligonucleotides were used for PCR: pcDNA-Dec $2 \Delta b$ : 5'-GACCG AATTAATGAATGCATT-3' and 5'-CTTGGTATCGTCT CGCTTCAA-3'; pcDNA-Dec2 $\triangle H L H$ : 5'-GCCTTAACTG AGCAGCAGCAT-3' and 5'-TCGTCTCTTCTTTTCTAT TAA-3'; pcDNA-Dec2 $\Delta O:$ 5'-CTGACGCCACAGGTGC CCTCC-3' and 5'-ATCCAAGTCGGCCTGGACCGG-3'. pcDNA-Dec2 $\Delta C$ (1-265) was constructed by digestion of pcDNA-mDec 2 with ApaI, followed by re-ligation. The expression vectors for Dec2 that fused with the GAL4 DNA binding domain (DBD) were constructed by subcloning various Dec2 cDNA fragments into the $\mathrm{BamHI}$ and $\mathrm{XbaI}$ sites of pBIND vector (Promega). For myc-tagged protein, fulllength Decl cDNA and various Dec2 cDNA fragments were subcloned into pcDNA3.1/Myc-His vector (Invitrogen). The flag-tagged $D e c 2$ construct was generated by subcloning fulllength Dec2 cDNA into the BglII and SalI sites of pFLAGCMV vector (Sigma). The bacterial expression vector for Dec2 that fused with glutathione S-transferase (GST) was constructed by subcloning full-length Dec2 cDNA into the EcoRI and HindIII sites of pET-41 vector (Novagen). Other expression constructs were generated by PCR followed by subcloning into pcDNA3.1 or pFLAG-CMV vectors. For muscle creatine kinase (CKM) reporter construct (pCKMpromoter-Luc), a 1.4-kbp 5'-upstream fragment (-1354 to $+37,+1$ indicates the translation initiation site) of mouse $C K M$ gene was amplified by PCR using a forward primer (5'-ACG CGTCAGCTGAGGTGCAAAAGGCTCCTGT-3') and a reverse primer (5'-CTCGAGTGAGTGTCTGTCTGTGCTG TGGA-3'), and ligated to the MluI and XhoI sites of pGL3Basic vector (Promega). For luciferase-reporter constructs containing three copies of the class A E-box, N-box, and class C E-box, the following pairs of oligonucleotides were annealed and cloned upstream of the thymidine kinase (TK) promoter of pTK-Luc vector (14): p3xE-A1-TK-Luc: 5'-CT AGTCCCAACACCTGCTGCCCCAACACCTGCTGCCCC AACACCTGCTGCCA-3' and 5'-TCGATGGCAGCAGGTG TTGGGGCAGCAGGTGTTGGGGCAGCAGGTGTTGGG A-3'; p3xN-TK-Luc: 5'-GTTTCACACGAGCCGTTCGTTT CACACGAGCCGTTCGTTTCACACGAGCCGTTC-3' and 5'-CGCGGAACGGCTCGTGTGAAACGAACGGCTCGT GTGAAACGAACGGCTCGTGTGAAACGTAC-3'; p3xEC-TK-Luc: 5'-CATCTGCACGCGACATCCCATCTGCAC GCGACATCCCATCTGCACGCGACATCC-3' and 5'-CGC
GGGATGTCGCGTGCAGATGGGATGTCGCGTGCAGA TGGGATGTCGCGTGCAGATGGTAC-3' . p3xE-B2-TKLuc, which contains three copies of the proximal class B E-box from the mouse Dec2 promoter, was described previously (14).

Luciferase assay. NIT3T3 and C2C12 cells were cultured in Dulbecco's modified Eagle's medium supplemented with $10 \%$ fetal bovine serum, penicillin (100 units/ml), streptomycin $(100 \mu \mathrm{g} / \mathrm{ml})$, and amphotericin $(250 \mathrm{ng} / \mathrm{ml})$. Cells were replated on 24 -well plates $\left(1 \times 10^{4}\right.$ cells/well), and the next day cells were transfected with the indicated combinations of expression vectors together with $20 \mathrm{ng}$ of a reporter plasmid using PolyFect Transfection Regent (Qiagen). The phRL-TK vector (2 ng) was cotransfected for normalization, and the total amount of DNA added to cells was adjusted to $0.2 \mu \mathrm{g} / \mathrm{well}$ by addition of pcDNA3.1 vector. Twenty-four hours after transfection, cells were harvested with passive lysis buffer to determine luciferase activity, using Dual-Luciferase reporter assay system (Promega).

GST pull-down assay. The BL21 strain of Escherichia coli was transformed with plasmid pET-41-Dec2 for expression of GST-Dec2 fusion protein or, as a control, pET-41 for expression of GST. GST pull-down assay was performed using purified GST and GST-Dec2 fusion proteins as described previously (15). $\left[{ }^{35} \mathrm{~S}\right]$ methionine-labeled proteins were synthesized in vitro using the TNT Quick coupled transcription/translation system (Promega).

Immunoprecipitation. COS-7 cells were transfected with expression vectors using PolyFect transfection regent. After $48 \mathrm{~h}$, cells were lysed in lysis buffer containing $20 \mathrm{mM}$ Tris$\mathrm{HCl}$ (pH 7.4), $150 \mathrm{mM} \mathrm{NaCl}, 10 \%$ glycerol, $0.5 \%$ Nonidet $\mathrm{P}-40$, and protease inhibitors (Roche). Lysates were incubated with anti-FLAG M2-Agarose affinity gel (Sigma) for $4 \mathrm{~h}$ at $4^{\circ} \mathrm{C}$. Immunoprecipitates were washed 3 times with buffer containing $50 \mathrm{mM}$ Tris- $\mathrm{HCl}$ (pH 7.4), $150 \mathrm{mM} \mathrm{NaCl}$, and then were eluted in SDS sample buffer. The immunoprecipitated proteins were analyzed by Western blotting.

Western blot analysis. After SDS-PAGE, proteins were transferred to polyvinylidene difluoride membrane (Millipore). Immunoblotting was performed with the appropriate antibodies according to standard protocols and detected using the 5-bromo-4-chloro-3-indolyl phosphate/nitro blue tetrazolium phosphatase substrate system. The following primary antibodies were used: Anti-myc-alkaline phosphatase (AP)-conjugated antibody (1:2000 dilution, Invitrogen), anti-FLAG M2 monoclonal antibody (1:5000 dilution, Sigma), and rabbit polyclonal antibody anti-Dec2 (1:2000 dilution). The Dec2-specific antibody was generated in rabbit against a synthetic peptide (Cys-Lys-Pro-Lys-Arg-Ser-Met-Lys-Arg-Asp-Asp-Thr-LysAsp) corresponding to a region in the $\mathrm{N}$-terminal region of the protein. The antibody was purified by affinity column chromatography. AP-conjugated secondary antibodies were used at a 1:2000 dilution.

Electrophoretic mobility shift assay (EMSA). EMSA was performed as described previously (14). For preparation of the probes, oligonucleotides were annealed and then end-labeled 
A

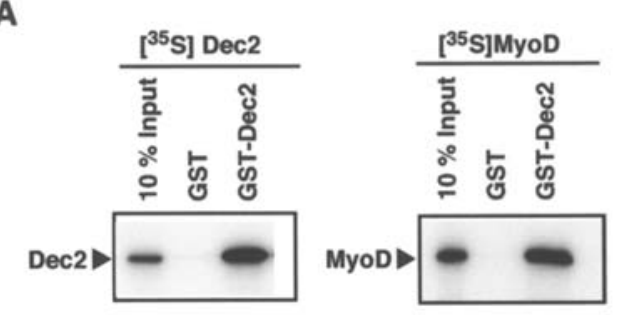

B

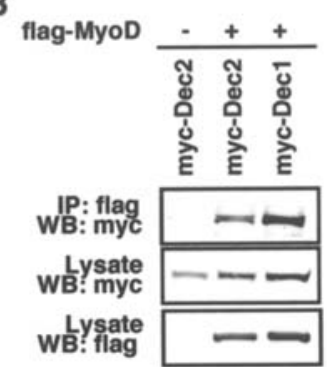

C

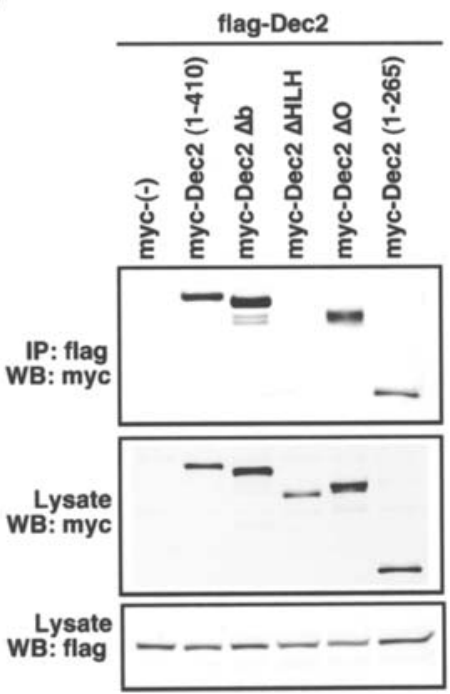

D

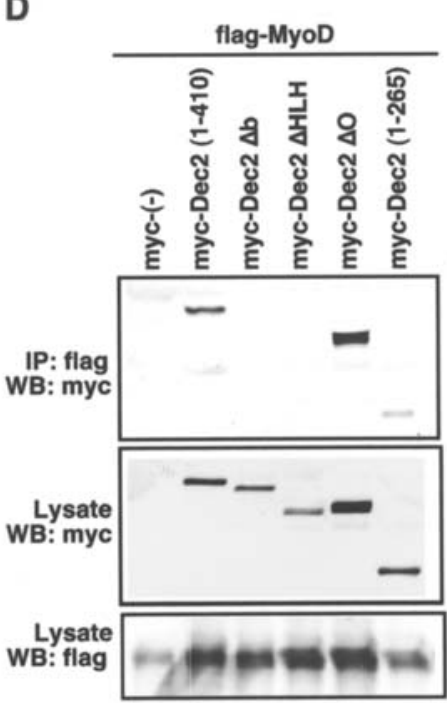

E

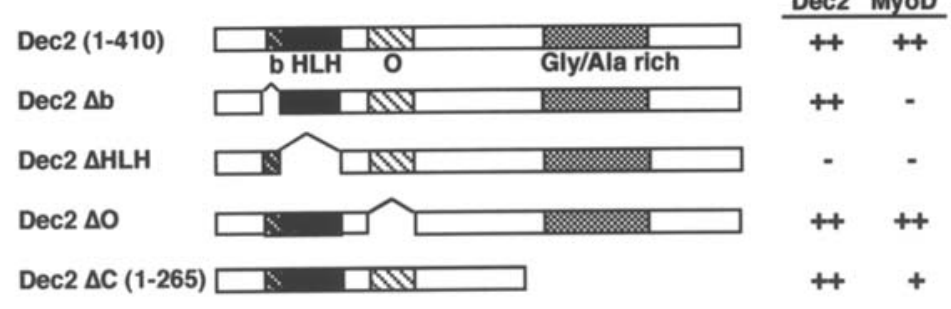

Figure 1. Homodimer and heterodimer formations of Dec proteins. (A) In vitro interaction of Dec2 with Dec2 and MyoD. GST and GST-Dec2 fusion proteins were purified from Escherichia coli and incubated with $\left[{ }^{35} \mathrm{~S}\right]$ methionine-labeled in vitro translated Dec2 or MyoD. Bound proteins were visualized by SDSPAGE and autoradiography. (B) Interaction of MyoD with Dec2 and Dec1 in mammalian cells. COS-7 cells were transfected with flag-tagged MyoD expression vector together with myc-tagged Dec 2 or myc-tagged Decl expression vector. Cell lysates were immunoprecipitated with anti-flag antibodies and immunoblotted with anti-myc antibodies. To confirm expression of Dec1, Dec2, and MyoD proteins, aliquots of total cell lysates were immunoblotted with the anti-flag or anti-myc antibodies. (C and D) Mapping of the Dec2 domains required for dimerization. COS-7 cells were transfected with the indicated myc-tagged Dec2 expression vectors together with flag-tagged Dec2 (C) or flag-tagged $M y o D$ (D) expression vector. Immunoprecipitation experiments were performed as described in panel B. (E) Schematic representation of full-length Dec2 (1-410) and its deletion mutants. b, basic region; HLH, helix-loop-helix domain; O, orange domain. Numbers indicate the positions of amino acids in the Dec2 protein.

with [32P]dCTP using DNA polymerase I Klenow fragment (Takara).

Real-time quantitative PCR. C2C12 cells plated on 6-well plates were transiently transfected as described above with $0.5 \mu \mathrm{g}$ each of the expression vector pcDNA-MyoD, pcDNAE47, pcDNA-Dec1, or pcDNA-Dec2 alone or the indicated combination. The total amount of DNA added was adjusted to $1.5 \mu \mathrm{g}$ /well by addition of pcDNA3.1 vector. Forty-eight hours after transfection, total RNA was prepared with RNeasy kit (Qiagen) and reverse-transcribed with ReverTra Ace (Toyobo) according to the manufacturer's instructions. Realtime PCR was performed as described previously (16). The synthesized first-strand cDNA was amplified using specific primers 5'-CATGGCGGCTACAAACCCA-3' and 5'-AGG TCGTCTCCACCCTTGAGGT-3' for mouse CKM. The amplified cDNA was quantified using 6FAM-ACAAGC ATAAGACCGACCTCAACCACGAG-TAMRA.

\section{Results}

Dimerizations of Dec proteins. We first examined whether Dec2 could form homodimer and heterodimer by GST pulldown assays. Both Dec2 and MyoD bound to GST-Dec2 but not to GST alone, indicating that Dec2 can interact directly with Dec2 and MyoD (Fig. 1A). We further investigated 
A

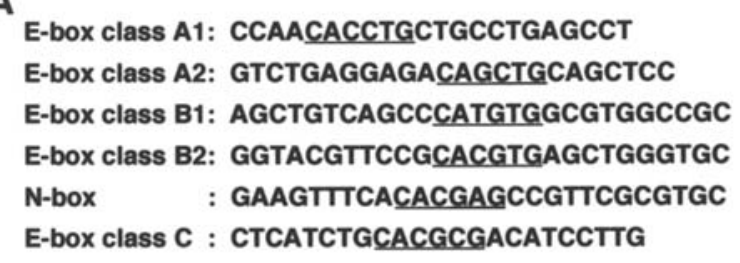

B

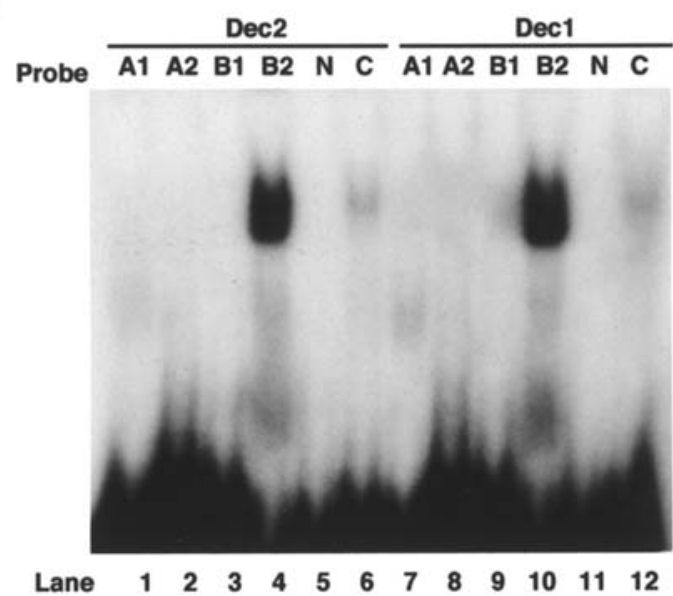

C

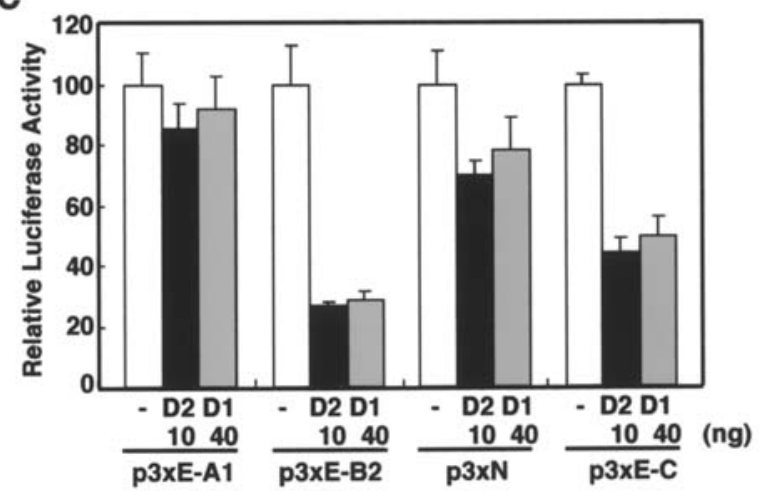

Figure 2. DNA binding and transcriptional repression activity of Dec protein homodimers. (A) Sense-strand sequences of double-stranded oligonucleotides used as probes in EMSA. Each oligonucleotide contains an E-box or N-box element responsive to bHLH transcription factors. The E-box and N-box sequences are underlined. E-boxA1, MyoD binding site from the $C K M$ promoter; E-boxA2, MyoD binding site from the Troponin I promoter; E-boxB1, USF1 and USF2 binding site from the Fatty acid synthase promoter; E-boxB2, Dec1 and Dec2 binding site from the Dec2 promoter; N-box, Hes1 binding site from the Hes1 promoter; E-boxC, Hes1 binding site from the Acid a-glucosidase promoter. (B) These radiolabeled oligonucleotide probes were incubated with in vitro translated Dec1 or Dec2 protein and subjected to EMSA. (C) Analysis of transcriptional repression by Dec1 and Dec2. NIH3T3 cells were transfected with the indicated reporter plasmids together with expression vectors for Dec1 (D1) or Dec2 (D2). Twenty-four hours after transfection, cell lysates were prepared and luciferase activity was assayed. The data presented are mean $\pm \mathrm{SD}$ for 4 independent experiments.

the interaction between Dec2 and MyoD in intact cells by co-immunoprecipitation assays. Efficient co-precipitation of Flag-MyoD with Myc-Dec2 was observed, and interaction of Dec1 with MyoD was also observed (Fig. 1B). However, it was not clear which regions of Dec2 were involved in the dimerizations. To address this issue, we generated a series of deletion mutations of Dec2. Deletion of the HLH domain of Dec2 (Dec2 $\Delta H L H)$ resulted in a complete loss of the interaction with full-length Dec2 (Fig. 1C and E). In contrast, all
A

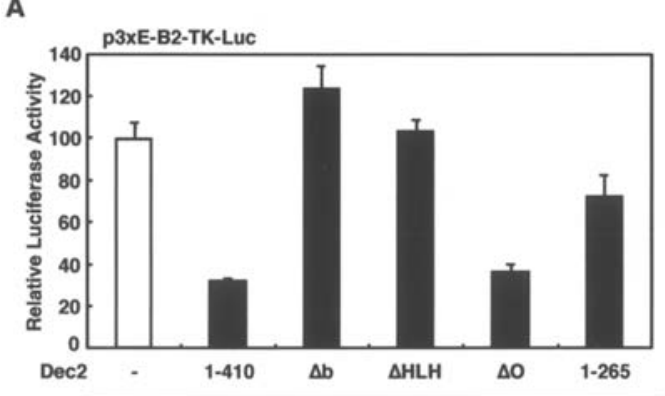

B

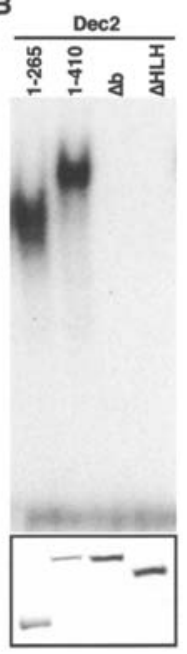

Figure 3. Mapping of functional domains required for Dec2-mediated transcriptional repression through direct DNA-binding. (A) Transcriptional activity of full-length Dec2 and deletion mutants through the CACGTG E-box element. NIH3T3 cells were transfected with the indicated Dec2 expression vectors together with the p3xE-B2-Luc reporter plasmid. The data presented are mean \pm SD for 4 independent experiments. (B) DNA binding activity of full-length Dec2 and deletion mutants. In vitro translated full-length and mutant Dec2 proteins were used in EMSA with radiolabeled double-stranded oligonucleotide E-boxB2. Shown below each lane are the results of Western blot analysis in which the corresponding samples were probed with anti-Dec2 antibody.

other deletion mutants (Dec2 $\Delta b$, Dec2 $2 \Delta \mathrm{O}$, and Dec $2 \Delta C$ ), as well as the wild-type Dec2, interacted efficiently with fulllength Dec2, indicating that Dec2 forms a homodimer through the HLH domain. We also tested the ability of Dec2 to heterodimerize with $\mathrm{MyoD}$ (Fig. 1D and E). Dec2 $\Delta \mathrm{O}$ interacted with MyoD, whereas Dec $2 \Delta b$ and Dec2 $\Delta H L H$ did not. In addition, deletion of the C-terminal domain of Dec2 partly reduced the interaction with $\mathrm{MyoD}$. These results are consistent with data showing that most bHLH proteins form a homodimer or heterodimer through the HLH domain. In addition, we found that the basic domain in Dec2 was also necessary for the Dec2-MyoD interaction.

DNA binding specificities of Dec proteins. We have shown that Dec1 and Dec2 proteins repress their own transcription by binding to CACGTG class B E-box element $(13,14)$, but bHLH-O proteins are well known to bind to other DNA sequences (2). We thus further examined the specificity of the protein-DNA interaction of Dec proteins. Dec1 and Dec2 proteins bound to the class B2 elements with high affinity (Fig. 2B, lanes 4 and 10), and also bound weakly to the class $\mathrm{C}$ elements (Fig. 2B, lanes 6 and 12), while no binding was detected with the A1, A2, B1, and N probes (Fig. 2B, lanes 1-3, 5, 7-9, and 11). To confirm that the Dec binding sites identified by EMSA are correlated with the transcriptional activity, we performed luciferase reporter assays. In transiently transfected NIH3T3 cells, both Dec1 and Dec2 repressed the promoter activity from the reporter containing class B2 elements, and to a lesser extent, class $\mathrm{C}$ elements, whereas they did not affect the transcriptional activity of the reporter containing class A1 elements (Fig. 2C). These results were consistent with the DNA binding activity of Dec proteins 
A

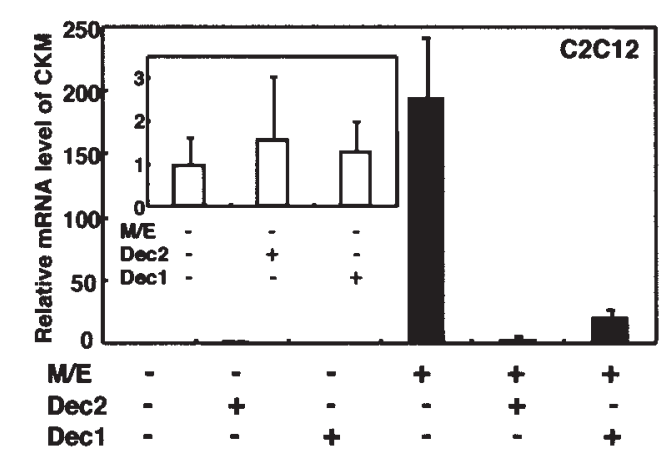

C

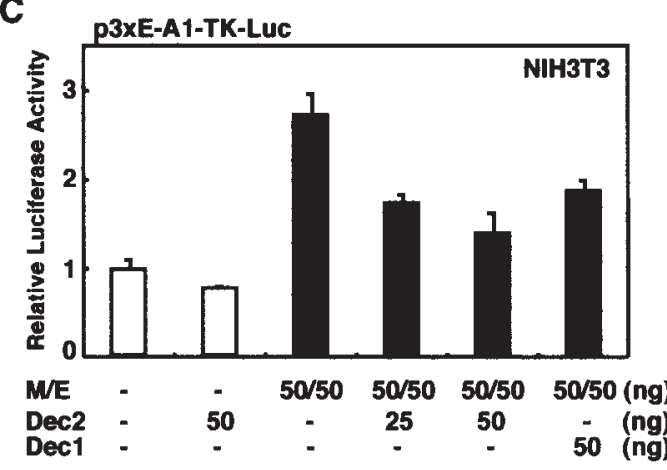

B

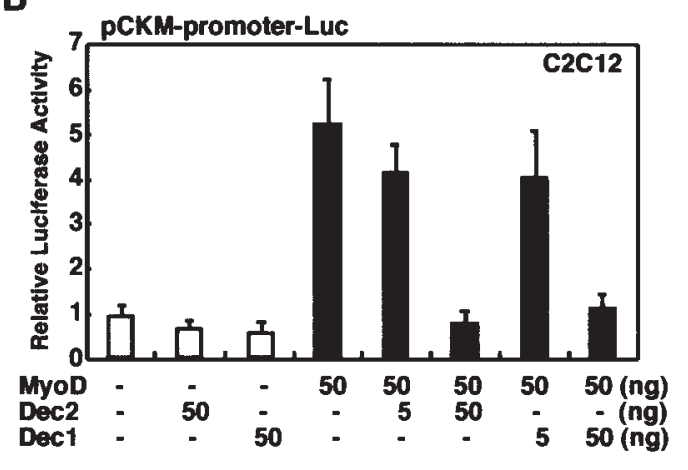

D

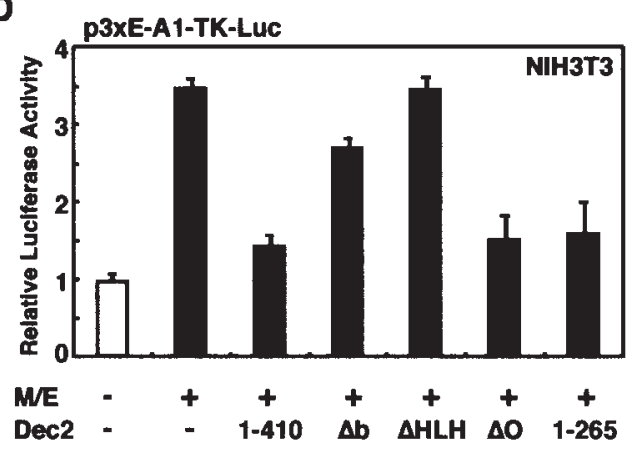

Figure 4. Inhibition of MyoD-mediated transactivation by Dec1 and Dec2. (A) Dec1 and Dec2 negatively regulate MyoD/E47-induced CKM mRNA expression. C2C12 cells were transfected with the indicated combinations of expression vectors for MyoD (M), E47 (E), Dec1, and Dec2. Forty-eight hours after transfection, total RNA was prepared to examine the expression levels of endogenous $C K M$ mRNA by real-time PCR analysis. $C K M$ expression patterns in the absence of MyoD and E47 are also shown in the inset with an expanded ordinate. (B) Dec1 and Dec2 repress MyoD-induced $C K M$ promoter activity. $\mathrm{C} 2 \mathrm{C} 12$ cells were transfected with the indicated expression vectors together with the pCKM-promoter-Luc reporter plasmid. (C) Dec1 and Dec2 repress E-box-dependent transactivation activity of MyoD/E47. NIH3T3 cells were transfected with the indicated expression vectors together with the p3xE-A1-Luc reporter plasmid. (D) The bHLH region in Dec2 is required to repress the transactivation of MyoD/E47. NIH3T3 cells were transfected with the indicated combinations of expression vectors for full-length $D e c 2, D e c 2$ deletion mutant, MyoD, and E47 together with the p3xE-A1-Luc reporter plasmid. The data presented are mean \pm SD for 4 independent experiments.

observed in Fig. 2A. Dec1 and Dec2 also repressed promoter activity weakly from the reporter containing N-box element, although no protein-DNA complexes were observed (Fig. 2A). This may be due to the instability of the complex in vitro under EMSA conditions.

Identification of functional domain of Dec2. To determine the functional domains in Dec2 required for transcriptional repression from CACGTG elements, we examined the effect of a series of deletions on the transcriptional activity of Dec2. Deletion of the Orange domain of Dec2 had little effect on the repression activity, but deletion of the HLH domain completely abolished the repression activity. This mutant did not form a homodimer (Fig. 1C), indicating that homodimer formation is necessary for the repression activity of Dec2 from CACGTG elements. In addition, deletion of the basic domain resulted in the inability of Dec2 to repress transcription, along with an increase in reporter activity over the control levels, suggesting that Dec $2 \Delta b$ acts as a dominant negative mutant to block the endogenous Dec2 function. Since the basic region in bHLH factors has been shown to mediate DNA binding (1), the inability of Dec $2 \Delta \mathrm{b}$ mutant to repress the promoter activity may be due to the inactivity of DNA binding. As expected, both Dec $2 \Delta b$ and Dec $2 \Delta H L H$ mutants failed to bind to the E-box sites, suggesting that the basic region and homodimer formation are necessary for the direct DNA binding activity of Dec2 (Fig. 3B). We also observed that truncation of the C-terminus of Dec2 decreased the repressive activity (Fig. 3A), even though this mutant did bind to the CACGTG E-box elements (Fig. 3B).

Decl and Dec2 repress MyoD-mediated CKM promoter activity. Since Dec1 and Dec2 formed not only a homodimer but also a heterodimer with MyoD (Fig. 1), we examined the effects of Dec proteins on expression of $C K M$, which is a physiological target gene for MyoD during muscle differentiation. Real-time PCR analysis showed that transfection of $M y o D$ and $E 47$ expression vectors induced an $\sim 200$-fold increase in $C K M$ mRNA expression in $\mathrm{C} 2 \mathrm{C} 12$ cells (Fig. 4A). The elevated expression level of $C K M$ mRNA was greatly reduced by cotransfection of Dec1 or Dec2, whereas Dec1 or Dec2 alone had little effect on $C K M$ mRNA expression (Fig. 4A, insert). We also examined the effects of Dec1 and Dec2 expression on the promoter activity of the CKM gene by reporter gene assay. MyoD up-regulated transcription of a reporter construct containing $1.3 \mathrm{~kb}$ of upstream sequence from the $C K M$ gene in $\mathrm{C} 2 \mathrm{C} 12$ cells, and this activation was efficiently reduced when $\mathrm{Dec} 1$ or Dec2 was cotransfected with MyoD (Fig. 4B).

The $C K M$ promoter contains a functional E-box element, which is essential for MyoD-induced gene expression in muscle differentiation (17). Therefore, we examined the effects 
A

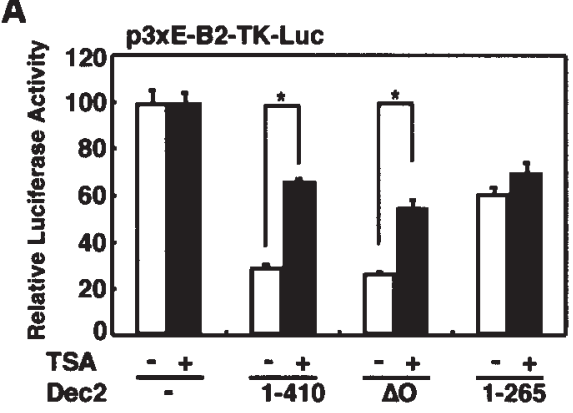

B

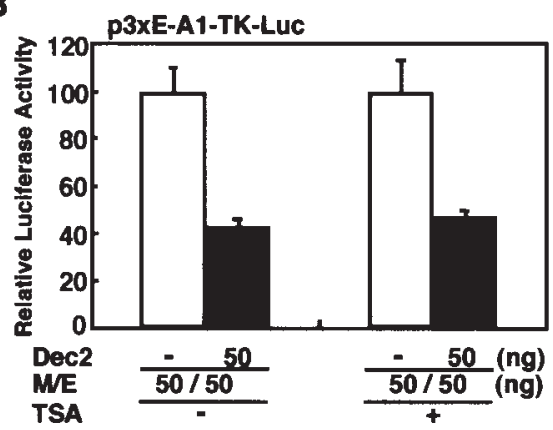

C
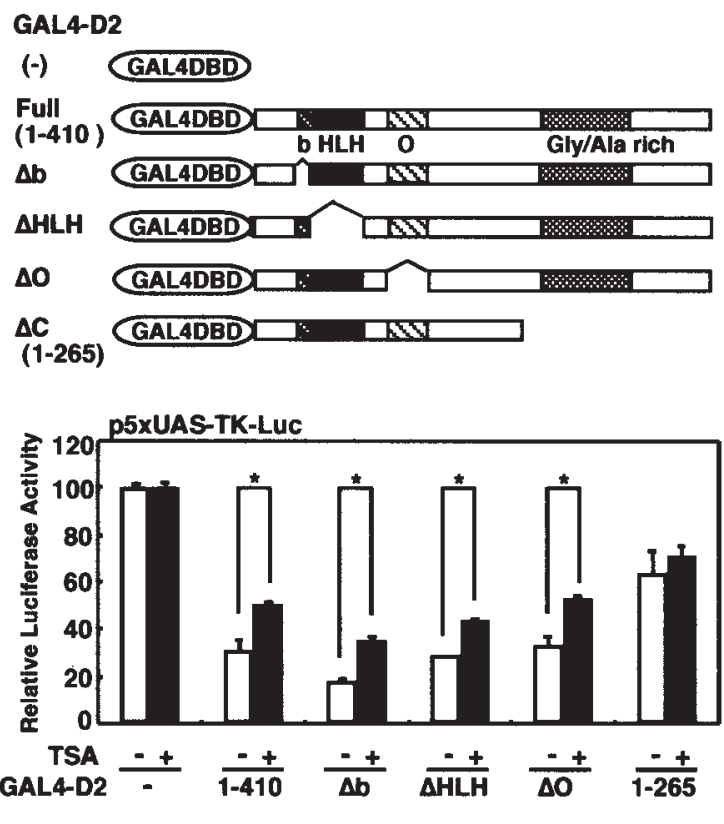

Figure 5. Effects of HDAC inhibitor on Dec2-mediated transcriptional repressions. NIH3T3 cells were transfected with the indicated combinations of expression vectors for Dec2, MyoD (M), and $E 47$ (E) together with the p3xE-B2-Luc (A) or p3xE-A1-Luc reporter plasmid (B). TSA (100 nM) was added $3 \mathrm{~h}$ after transfection, and then incubation was continued for $21 \mathrm{~h}$. Luciferase activity is expressed as percent in the absence of Dec2. (C) NIH3T3 cells were transfected with the indicated GAL4DBD-Dec2 expression vectors (GAL4-D2) together with the luciferase reporter plasmid containing the 5x GAL4 binding site (p5xUAS-TK-Luc). TSA $(100 \mathrm{nM})$ was added $3 \mathrm{~h}$ after transfection, and then incubation was continued for $21 \mathrm{~h}$. Luciferase activity is expressed as percent in the absence of Dec2. The data presented are mean \pm SD for 4 independent experiments. ${ }^{*} \mathrm{P}<0.02$.

A

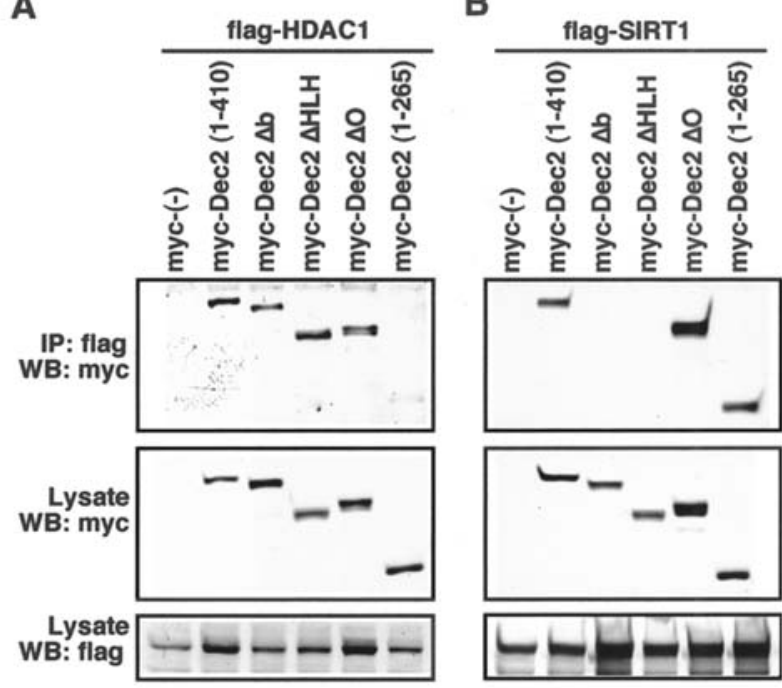

Figure 6. Interaction of Dec2 with HDACs. COS-7 cells were transfected with the indicated myc-tagged Dec2 expression vectors together with flagtagged HDAC1 (A) or flag-tagged SIRT1 (B) expression vector. Cell lysates were immunoprecipitated with anti-flag antibodies and immunoblotted with anti-myc antibodies. To confirm expression of Dec2, HDAC1, and SIRT1 proteins, aliquots of total cell lysates were immunoblotted with the anti-flag or anti-myc antibodies.

of Dec1 and Dec2 on E-box-dependent transactivation activity of MyoD. Cotransfection of MyoD with E47 activated transcription of the reporter gene p3xE-A1-TK-Luc containing three repeats of the E-box in the $C K M$ enhancer. Both Dec1 and Dec2 repressed the MyoD-mediated transactivation, although each alone had little effect on the transcriptional activity (Fig. 4C). Deletion of the HLH domain completely abolished the repression activity, and deletion of the basic domain resulted in a $65 \%$ reduction in repression activity (Fig. 4D). Taken together with the interaction data (Fig. 1D), this suggests that interaction of the bHLH domain in Dec2 with MyoD is crucial for the ability of Dec2 to repress MyoDmediated transactivation.

Effects of trichostatin A (TSA) on Dec2-mediated transcriptional repressions. Numerous transcriptional repressors associate with histone deacetylase (HDAC) and exert repression by recruitment of HDAC complexes to the appropriate promoter sites. Treatment of NIH3T3 cells with $100 \mathrm{nM}$ TSA, an inhibitor of class I and II HDACs, partially relieved the Dec2-mediated transcriptional repression from CACGTG elements. In addition, transcriptional repression by Dec $2 \Delta \mathrm{O}$ mutant was also inhibited by the TSA treatment, but transcriptional repression by Dec $2 \Delta \mathrm{C}$ mutant was not affected by the TSA treatment (Fig. 5A). Therefore, Dec2-mediated repression of CACGTG E-box containing promoter is probably partially dependent on the activity of TSA-sensitive HDACs, and the C-terminus of Dec2 is required for the HDACdependent pathway. However, treatment with TSA had little effect on the ability of Dec2 to repress MyoD-mediated transactivation from CACCTG elements (Fig. 5B). The difference in sensitivity to TSA was not due to a difference in the amount of transfected Dec2 expression construct, because TSA inhibited Dec2-mediated transcriptional repression from CACGTG elements when the same amount of Dec2 expression 
vector (50 ng) was transfected (data not shown). The findings suggest that Dec2 represses MyoD-mediated transactivation class I and II HDACs-independently.

Using a GAL4 fusion system, we further examined whether binding of Dec2 to a particular promoter would inhibit transcription through the recruitment of HDAC activity (Fig. 5C). As expected, Gal4-Dec2 full-length (1-410) showed a strong transcriptional repression activity, and the repression was partially relieved by TSA treatment. Similarly, several Gal4fused Dec2 mutants (Gal4-D2 $\Delta b$, Gal4-D2 $\Delta$ HLH, Gal4$\mathrm{D} 2 \Delta \mathrm{O})$ also repressed transcription and were sensitive to TSA. In contrast, truncation of the C-terminus reduced Dec2 transcriptional repression activity by $50 \%$, and the repression mediated by Gal4-D2 (1-265) was insensitive to TSA, indicating the presence of a repressor domain within the Cterminal region, which may involve recruitment of HDACs.

Interaction of Dec2 with HDAC1 and SIRT1. To confirm the involvement of HDACs in Dec2-mediated transcriptional repression, we investigated the interaction of Dec2 with HDAC1 and SIRT1, which are class I and III HDACs, respectively. Immunoprecipitation analysis revealed that fulllength Dec2, Dec2 $\Delta b$, Dec $2 \Delta H L H$, and Dec $2 \Delta \mathrm{O}$ interact with HDAC1, whereas Dec $2 \Delta \mathrm{C}$ did not (Fig. 6A). These results indicate that Dec2 associates with HDAC1 through its C-terminal region in vivo. In addition, Dec2 interacted with SIRT1 through its bHLH region (Fig. 6B).

\section{Discussion}

The present study demonstrated that Dec1 and Dec2 function as transcriptional repressors through two different mechanisms. i) Homodimers of Dec proteins bind directly to specific DNA sites and repress transcription of target genes. The repression is partly mediated by the recruitment of corepressor components including HDAC1. ii) Dec can form heterodimers with MyoD and inhibit MyoD-dependent transactivation, probably by preventing MyoD/E-protein from binding to DNA. TSA had no effect on the repression activity of Dec2; therefore, it is likely that HDAC1 is not involved in the ability of Dec2 to repress MyoD-mediated transactivation. Deletion analysis showed that both the basic and HLH domains in Dec2 are essential for the two repression mechanisms, and our results also revealed that Dec1 and Dec2 have similar DNA binding specificities and transcriptional repression activities, which suggests some functional redundancy between these family members.

Previous studies with bHLH-O proteins demonstrated that each subfamily possesses intrinsic transcriptional repressor domains and employs a different mechanism to repress transcription. Hes1 represses transcription by recruiting a TLE/ Groucho corepressor through the WRPW motif in the Cterminus (2), which is conserved among the Hes family. Hey1 and Hey2, members of the Hey family, share similar motifs, YRPW and YQPW, respectively, in the C-terminus. However, a recent study demonstrated that these motifs are not essential for their transcriptional repression (18). The repression activity of Hey1 is mediated by the bHLH region, which is involved in the recruitment of the complex containing mSin $3 \mathrm{~A}, \mathrm{~N}-\mathrm{CoR}$, and HDAC1. On the other hand, Dec and Helt family members lack the WRPW motif, and both the Orange domain and the $\mathrm{C}$-terminal region in Helt are required for their repression activity (3). The Orange domain of Hes 1 is also required for its repressor activity. However, our mutagenesis studies showed that the $\mathrm{C}$-terminal region but not the Orange domain of Dec2 is essential for its repression activity. Thus, the mechanism of Dec-mediated active transcriptional repression differs from those of other bHLH-O family members.

MyoD activity is negatively regulated by several transcriptional regulators, including Id, MyoR, Mist1 and Dermo-1, through protein-protein interactions (19). Many of these proteins form inactive heterodimers with MyoD and/or Eprotein and inhibit their function. Recently, Dec2 has been reported to inhibit MyoD-induced muscle differentiation. Dec2 can associate with MyoD and attenuate the ability of the MyoD/E47 heterodimer to bind DNA (10). Our present study extended these findings, and showed that both the basic and $\mathrm{HLH}$ domains in Dec2 are required for interaction with MyoD and inhibition of MyoD transcriptional activity. We also showed that similar to Dec2, Dec1 represses the transcriptional activity of MyoD. Furthermore, we found that the bHLH in Dec2 were also required for interaction with Sirt1. It has been reported that SIRT1 deacetylates MyoD and represses its activity. Overexpression of SIRT1 inhibited muscle gene expression and differentiation of myoblasts into multinucleated myocytes, and its deacetylase activity is essential for this inhibition (20). SIRT1 may be involved in Dec2-mediated repression for MyoD activity.

In conclusion, Dec1 and Dec2 negatively regulated transcriptional activity not only by direct binding to target genes but also by inhibiting MyoD activity. Dec1 also interacts with other bHLH proteins, such as USF and Bmal1, and inhibits their activities $(15,21)$. Thus, Dec proteins form multiple dimer combinations with other bHLH proteins and mediate various gene expressions by modulating their transcriptional activities.

\section{Acknowledgements}

This study was supported by grants-in-aid for science from the Ministry of Education, Culture, Sport, Science and Technology of Japan. We wish to thank the Research Center for Molecular Medicine, Faculty of Medicine, Hiroshima University for the use of their facilities.

\section{References}

1. Murre C, Bain G, van Dijk MA, Engel I, Furnari BA, Massari ME, Matthews JR, Quong MW, Rivera RR and Stuiver MH: Structure and function of helix-loop-helix proteins. Biochim Biophys Acta 1218: 129-135, 1994.

2. Davis RL and Turner DL: Vertebrate hairy and Enhancer of split related proteins: transcriptional repressors regulating cellular differentiation and embryonic patterning. Oncogene 20: 8342-8357, 2001

3. Nakatani T, Mizuhara E, Minaki Y, Sakamoto Y and Ono Y: Helt, a novel basic-helix-loop-helix transcriptional repressor expressed in the developing central nervous system. J Biol Chem 279: 16356-16367, 2004.

4. Shen M, Kawamoto T, Yan W, Nakamasu K, Tamagami M, Koyano Y, Noshiro M and Kato Y: Molecular characterization of the novel basic helix-loop-helix protein DEC1 expressed in differentiated human embryo chondrocytes. Biochem Biophys Res Commun 236: 294-298, 1997. 
5. Rossner MJ, Dorr J, Gass P, Schwab MH and Nave KA: SHARPs: mammalian enhancer-of-split- and hairy-related proteins coupled to neuronal stimulation. Mol Cell Neurosci 9: 460-475, 1997.

6. Boudjelal M, Taneja R, Matsubara S, Bouillet P, Dolle P and Chambon P: Overexpression of Stra13, a novel retinoic acidinducible gene of the basic helix-loop-helix family, inhibits mesodermal and promotes neuronal differentiation of P19 cells. Genes Dev 11: 2052-2065, 1997.

7. Fujimoto K, Shen M, Noshiro M, Matsubara K, Shingu S, Honda K, Yoshida E, Suardita K, Matsuda Y and Kato Y: Molecular cloning and characterization of DEC2, a new member of basic helix-loop-helix proteins. Biochem Biophys Res Commun 280: 164-171, 2001.

8. Shen M, Yoshida E, Yan W, Kawamoto T, Suardita K, Koyano Y, Fujimoto K, Noshiro M and Kato Y: Basic helix-loop-helix protein DEC1 promotes chondrocyte differentiation at the early and terminal stages. J Biol Chem 277: 50112-50120, 2002.

9. Yun Z, Maecker HL, Johnson RS and Giaccia AJ: Inhibition of PPAR gamma 2 gene expression by the HIF-1-regulated gene DEC1/Stra13: a mechanism for regulation of adipogenesis by hypoxia. Dev Cell 2: 331-341, 2002.

10. Azmi S, Ozog A and Taneja R: Sharp-1/DEC2 inhibits skeletal muscle differentiation through repression of myogenic transcription factors. J Biol Chem 279: 52643-52652, 2004

11. Sun H, Lu B, Li RQ, Flavell RA and Taneja R: Defective T cell activation and autoimmune disorder in Stra13-deficient mice. Nat Immunol 2: 1040-1047, 2001.

12. Honma S, Kawamoto T, Takagi Y, Fujimoto K, Noshiro M, Sato F, Kato Y and Honma K: Dec1 and Dec2 are regulators of the mammalian molecular clock. Nature 419: 841-844, 2002.

13. Kawamoto T, Noshiro M, Sato F, Maemura K, Takeda N, Nagai R, Iwata T, Fujimoto K, Furukawa M, Miyazaki K, Honma S, Honma K and Kato Y: A novel autofeedback loop of Dec1 transcription involved in circadian rhythm regulation. Biochem Biophys Res Commun 313: 117-124, 2004.
14. Hamaguchi H, Fujimoto K, Kawamoto T, Noshiro M, Takeda N, Maemura K, Nagai R, Furukawa M, Honma S, Honma K, Kurihara $\mathrm{H}$ and Kato Y: Expression of the gene for Dec2, a basic helix-loop-helix transcription factor, is regulated by a molecular clock system. Biochem J 382: 43-50, 2004.

15. Sato F, Kawamoto T, Fujimoto K, Noshiro M, Honda KK, Honma S, Honma $\mathrm{K}$ and Kato Y: Functional analysis of the basic helix-loop-helix transcription factor DEC1 in circadian regulation. Interaction with BMAL1. Eur J Biochem 271: 4409-4419, 2004.

16. Suardita K, Fujimoto K, Oda R, Shimazu A, Miyazaki K, Kawamoto $\mathrm{T}$ and Kato Y: Effects of overexpression of membrane-bound transferrin-like protein (MTf) on chondrogenic differentiation in vitro. J Biol Chem 277: 48579-48586, 2002.

17. Amacher SL, Buskin JN and Hauschka SD: Multiple regulatory elements contribute differentially to muscle creatine kinase enhancer activity in skeletal and cardiac muscle. Mol Cell Biol 13: 2753-2764, 1993

18. Iso T, Sartorelli V, Poizat C, Iezzi S, Wu HY, Chung G, Kedes L and Hamamori Y: HERP, a novel heterodimer partner of $\mathrm{HES} / \mathrm{E}(\mathrm{spl})$ in Notch signaling. Mol Cell Biol 21: 6080-6089, 2001.

19. Puri PL and Sartorelli V: Regulation of muscle regulatory factors by DNA-binding, interacting proteins, and post-transcriptional modifications. J Cell Physiol 185: 155-173, 2000.

20. Fulco M, Schiltz RL, Iezzi S, King MT, Zhao P, Kashiwaya Y, Hoffman E, Veech RL and Sartorelli V: Sir2 regulates skeletal muscle differentiation as a potential sensor of the redox state. Mol Cell 12: 51-62, 2003.

21. Dhar M and Taneja R: Cross-regulatory interaction between Stra13 and USF results in functional antagonism. Oncogene 20: 4750-4756, 2001 